\title{
Implementación del Sistema de Innovación Agropecuaria Local en el municipio Sancti Spíritus, Cuba
}

Implementation of the Local Agricultural Innovation System in the municipality of Sancti Spíritus, Cuba

Implementação do sistema de inovação agropecuária local no município Sancti Spíritus, Cuba

\author{
Rosabell Pérez Gutiérrez / Universidad de Sancti Spíritus, Cuba / rosabell@uniss.edu.cu \\ Alena Medina Echevarría / Universidad de Sancti Spíritus, Cuba / alena@uniss.edu.cu \\ Dayana Salabarría Cruz / Universidad de Sancti Spíritus, Cuba / dayanasc@uniss.edu.cu \\ Nelson León Orellana / Universidad de Sancti Spíritus, Cuba / orellana@uniss.edu.cu
}

Recibido: 20/11/2020 Aceptado: 16/3/2021 Publicado: 15/6/2021

\section{RESUMEN}

El despliegue de experiencias innovadoras a nivel local permite la creación de capacidades en favor del desarrollo sostenible. En la presente investigación se propuso diseñar un sistema de acciones para la institucionalización del Sistema de Innovación Agropecuaria Local en el municipio de Sancti Spíritus, basado en un estudio de las particularidades del sector agropecuario. Para ello, se asumió una metodología mixta y se emplearon como técnicas: la entrevista en profundidad, la observación participante y el análisis documental. Los instrumentos aplicados permitieron identificar las fortalezas y debilidades del contexto agropecuario y la innovación en el territorio espirituano, durante el periodo 2018-2020, y sus resultados reafirmaron la necesidad de dinamizar los procesos innovadores mediante un plan de acción, donde los actores sociales fueron el recurso fundamental. A raíz, la propuesta de acciones diseñada permite el diálogo, la participación y la retroalimentación de los saberes teóricos y prácticos, y reconoce a los productores como los verdaderos protagonistas del desarrollo en el sector.

Palabras clave: desarrollo local, innovación agraria, sector agropecuario, sistema de acciones 


\begin{abstract}
The deployment of innovative experiences at the local level allows the creation of capacities for sustainable development. In this research, we proposed to design a system of actions for the institutionalization of the Local Agricultural Innovation System in the Sancti Spíritus municipality, based on a study of the particularities of the agricultural sector. For this, a mixed methodology was assumed and the following techniques were used: in-depth interview, participant observation and documentary analysis. The applied instruments made possible to identify the strengths and weaknesses of the agricultural and innovation context in the Sancti Spiritus territory, during the 2018-2020 period, and their results reaffirmed the need to stimulate the innovative processes through an action plan, where the social actors were the fundamental resource. As a result, the proposed actions designed allow dialogue, participation and feedback on theoretical and practical knowledge, and recognize producers as the true protagonists of development in the sector.
\end{abstract}

Keywords: action system, agricultural innovation, agricultural sector, local development

\title{
RESUMO
}

A implantação de experiências inovadoras em nível local permite a criação de capacidade para o desenvolvimento sustentável. Esta pesquisa teve como objetivo desenhar um sistema de ações para a institucionalização do Sistema de Inovação Agropecuária Local no município de Sancti Spíritus, a partir de um estudo das particularidades do setor agropecuário. Para isso, foi assumida uma metodologia mista e foram utilizadas as seguintes técnicas: entrevista em profundidade, a observação participante e análise documental. Os instrumentos aplicados permitiram identificar os pontos fortes e fracos do contexto agropecuário e da inovação no território durante o período 2018-2020, onde os resultados reafirmaram a necessidade de estimular os processos inovadores através de um plano de ação, onde os atores sociais foram o recurso fundamental. Com isso, a proposta de ação desenhada permite o diálogo, a participação e a retroalimentação de conhecimentos teóricos e práticos, e reconhece os produtores como verdadeiros protagonistas do desenvolvimento do setor.

Palavras chave: desenvolvimento local, inovação agrícola, setor agropecuário, sistema de ações INTRODUCCIÓN

El desarrollo emerge como desafío para la construcción de un orden social humanizador. Aciertos y contradicciones inciden en sus esencias, apropiaciones, prácticas e impactos. Como parte de su abordaje crítico, adquiere renovados sentidos éticos y compromisos políticos, en diálogo y confrontación entre los sujetos sociales implicados, en disputa con sus imaginarios y percepciones; lo que representa un despertar de los procesos emancipatorios que sostienen la idea del 
cambio. Sus visiones y apuestas requieren, también, una reflexión más crítica y profunda para lograr la construcción de sociedades sostenibles con la participación de todos (Stewart-Santos et al., 2020).

Para Cuba, el actual contexto político ofrece nuevas concepciones y oportunidades a los procesos de descentralización y autonomía municipal definidos en los documentos rectores de la política nacional. De manera especial la Constitución de la República, refrendada en el 2019, y la Política de Desarrollo Territorial, aprobada en el 2020, abren la puerta a procesos más democráticos, autónomos y participativos con la implementación de las estrategias de desarrollo municipal.

Consecuentemente, en el escenario cubano, el desarrollo local muestra un amplio campo de investigaciones con testimonios que trascienden el universo académico, y se posicionan como tema prioritario en las agendas políticas de gobiernos y entidades de la ciencia, la tecnología y la innovación. Desde esta perspectiva, se concibe como un proceso endógeno construido de abajo hacia arriba; que estimula la articulación entre los diversos actores de los procesos de transformación a escala micro; y que procura entornos donde las personas desarrollen capacidades para solucionar problemas concretos de territorios y comunidades, de acuerdo con sus necesidades e intereses. Para ello, favorece la cooperación, el diálogo de saberes, el compromiso colectivo y la participación democrática en la toma de decisiones.

Los arreglos y sistemas productivos e innovativos locales representan, fundamentalmente, un cuadro de referencias para comprender los procesos de generación, difusión y utilización del conocimiento, y los de la dinámica productiva e innovadora (Garcés et al., 2020). Se trata de asumir la innovación como elemento dinamizador del desarrollo local desde las relaciones entre los gobiernos municipales, los consejos populares, los actores del territorio, las empresas, los procesos comunitarios y las prácticas socioculturales (Fajardo et al., 2013); pues como bien plantean Núñez y Alcázar (2016), de esta premisa depende su máximo aprovechamiento.

El desarrollo de la agricultura y la producción de alimentos se posicionan como temas prioritarios en la agenda del municipio Sancti Spíritus. Para hacer frente a este propósito, se implementan proyectos orientados a perfeccionar el sistema agroproductivo con la creación y el fortalecimiento de capacidades innovadoras, en función del desarrollo sostenible; entre ellos, el Proyecto de Innovación Agropecuaria Local, coordinado por el Instituto Nacional de Ciencias Agrícolas desde hace 15 años, propone consolidar sus resultados a través de su incidencia en las políticas públicas y la difusión de buenas prácticas, con la institucionalización de los Sistemas de Innovación Agropecuaria Local (SIAL).

En ese marco, los SIAL persiguen promover y sistematizar un conjunto de prácticas y metodologías con la participación activa de los productores, en los denominados Grupos de Innovación Agropecuaria Local, para encontrar soluciones innovadoras en la agricultura a partir del involucramiento de los actores locales (Ortiz et al., 2016); por ello, constituyen una propuesta sobre cómo instrumentar un sistema de gestión del 
conocimiento y el desarrollo en municipios cubanos, de manera horizontal y participativa (Romero et al., 2018). En paralelo, asumen un modelo de innovación amplio, teóricopráctico, que se basa en la formación de capacidades y la consolidación de prácticas de gestión de la innovación, bajo la filosofía: haciendo-usando-interactuando.

En Sancti Spíritus, el Proyecto de Innovación Agropecuaria Local prioriza la institucionalización del SIAL a través de la creación de las plataformas multiactorales de gestión, consideradas espacios idóneos para el debate y la reflexión de los problemas del sector agropecuario. Tal reconocimiento es imprescindible para el desarrollo integral (económico, político, social) del municipio espirituano, si se tiene en cuenta la necesidad de impulsar la gestión compartida entre el gobierno y los actores locales, desde el prisma de la agrodiversidad, como estrategia a favor de la seguridad y la soberanía alimentarias.

No obstante, a pesar de los múltiples esfuerzos por parte de la coordinación y el equipo de trabajo del Proyecto de Innovación Agropecuaria Local, aún existen grandes limitaciones para alcanzar tales propósitos, debido a la falta de conocimiento y sensibilidad de los actores en relación a la propuesta; la inestabilidad de los funcionarios y directivos que integran el sistema del Poder Popular Municipal y el Consejo de Administración Municipal; el surgimiento de barreras para la institución de las plataformas multiactorales de gestión; la escasa articulación entre los actores locales miembros de los Grupos de Innovación Agropecuaria Local; y la limitada capacitación en cuanto a las oportunidades que ofrece el SIAL para la gestión del desarrollo local, fundamentalmente.

Como puede evidenciarse, la ideología, la forma de organización social y las condicionantes socioeconómicas (Arzola et al., 2017) forman parte de los factores que inciden en el desarrollo agroproductivo de la provincia. Sobre esta base, la presente investigación persiguió diseñar un sistema de acciones para la institucionalización del SIAL en el municipio Sancti Spíritus, basado en un estudio de las particularidades del sector agropecuario.

Teniendo en cuenta la pluralidad y diversidad de individuos y grupos que se definen como actores en el escenario rural local, resulta vital entender el valor de sus aportes en la concepción de las estrategias de desarrollo, al ser ellos los verdaderos protagonistas del cambio. De esta forma, cualquier alternativa a considerar para institucionalizar los SIAL debe partir de una descripción de las particularidades del contexto agropecuario y de la innovación en el municipio, para profundizar en las interrelaciones del sistema de conocimientos populares agrícolas y los elementos que definen el desarrollo rural a nivel local.

\section{MATERIALES Y MÉTODOS}

Se asumió una metodología mixta al considerarse la utilización de métodos y técnicas tanto de tipo cualitativo como cuantitativo. Desde la definición del tema de investigación, la fenomenología destacó la importancia de la dimensión cultural de la realidad social, a partir de la resignificación e interpretación de cada uno de los actores y del fenómeno a investigar.

Se aplicó el análisis documental entre las técnicas e instrumentos para el trabajo de campo, lo que permitió sistematizar 
los elementos legislativos, organizativos y estratégicos que estructuran el sistema agropecuario en Cuba, con particular énfasis en el contexto objeto de análisis. Se consultaron con este fin: la Estrategia de Desarrollo de Sancti Spíritus (2015-2020); el Programa de Desarrollo Agropecuario del municipio de Sancti Spíritus (2015-2030); el Plan de Capacitación (2015-2018), del Ministerio de la Agricultura; el Anuario Estadístico de Cuba (Oficina Nacional de Estadísticas e Información, 2019); y las relatorías de las actividades del Proyecto de Innovación Agropecuaria Local.

En aras de conocer las particularidades de Sancti Spíritus en la gestión del desarrollo local y agropecuario, se realizaron entrevistas en profundidad a actores vinculados a la gestión del Poder Popular Municipal, así como a aquellos cuyo desempeño laboral tuviera un fuerte protagonismo dentro del sector; entre ellos: la coordinadora del fórum en la Asamblea Municipal del Poder Popular y el vicepresidente que allí atiende el desarrollo local; el delegado y la subdelegada municipal del Ministerio de la Agricultura; el especialista de cuadros de la Delegación Provincial del Ministerio de la Agricultura; el titular de la Dirección Provincial de Suelos y Fertilizantes; el coordinador de la Escuela de Capacitación y vicepresidente de la Asociación Cubana de Técnicos Agrícolas Forestales; el coordinador de proyectos en la Asociación Nacional de Agricultores Pequeños; y el coordinador provincial e integrantes del Proyecto de Innovación Agropecuaria Local.

También se empleó la observación participante en actividades agrícolas, asambleas de las cooperativas y en los recorridos por las fincas de los productores. Finalmente, se realizó la triangulación metodológica para contrastar la información resultante de los diferentes instrumentos aplicados. Para esta investigación, la selección de la muestra se realizó a partir de un muestreo no probabilístico (guiado), donde los sujetos se eligen de forma intencionada, de acuerdo con los criterios establecidos por el investigador (Hernández et al., 2014).

\section{RESULTADOS Y DISCUSIÓN}

\section{Caracterización del contexto agropecuario en el municipio Sancti Spíritus}

El municipio Sancti Spíritus cuenta con 53 asentamientos poblacionales, de ellos cuatro urbanos y 49 rurales, distribuidos en 13 consejos populares y 144 circunscripciones. La densidad de población es de $122.37 \mathrm{hab} . / \mathrm{km}^{2}$, aunque existe una distribución desigual entre la cabecera municipal y los consejos populares ubicados en sus zonas rurales. En este sentido, los asentamientos urbanos muestran un acelerado crecimiento poblacional, mientras que los rurales exhiben un notable estancamiento o decrecimiento, principalmente en los de menor densidad poblacional.

Por su parte, la población en edad laboral vinculada al sector agropecuario es de 10546, de ellos: 8310 hombres y 2236 mujeres; lo cual representa el $21.1 \%$ de este grupo poblacional. Aun cuando el Ministerio de la Agricultura y las organizaciones sociales vinculadas a él incorporan objetivos de equidad social o de género, y continúan los esfuerzos por acortar la brecha y transformar la percepción de los roles históricamente atribuidos a hombres y mujeres, en la práctica cotidiana se expresan 
inequidades: la mayor parte de la fuerza de trabajo de las unidades de producción del sector agropecuario, además de proceder de los asentamientos rurales, está integrada principalmente por hombres.

Figura 1. Fuerza de trabajo en el municipio
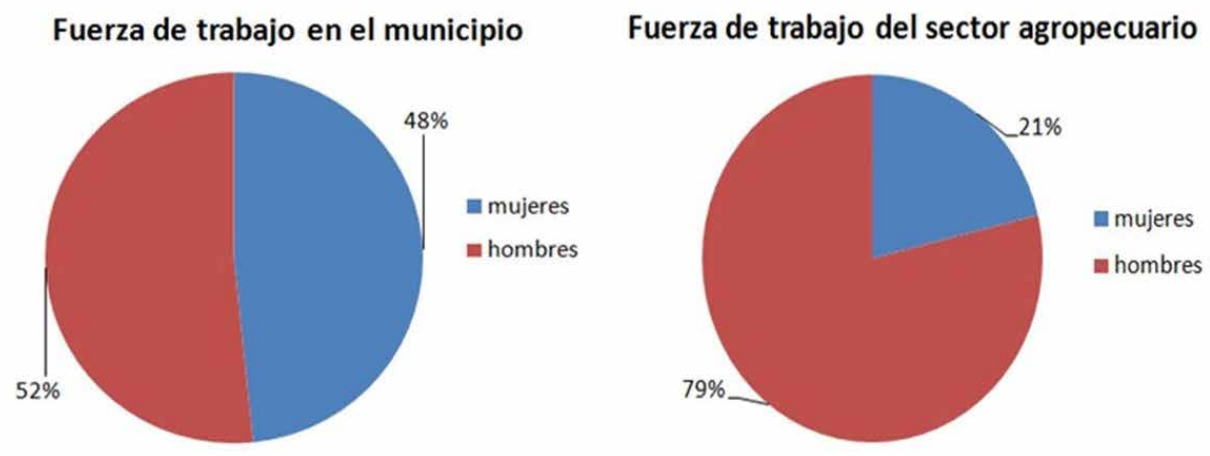

Fuente: Elaboración propia

La Asamblea Municipal del Poder Popular de Sancti Spíritus tiene la responsabilidad de implementar políticas y generar condiciones favorables para el desarrollo productivo y el mejoramiento de las condiciones de vida de la población. Una de sus principales herramientas para cumplir tal encargo a escala local es la Estrategia de Desarrollo de Sancti Spíritus (2015-2020), la cual constituye una guía de acción social basada en las características, necesidades y potencialidades del territorio, y que precisa de una activa participación e integración entre los actores para promover una dinámica de cambio.

Dentro de las cuatro líneas estratégicas que perfila dicha herramienta para otorgar prioridades y organizar las acciones propuestas, el desarrollo de la agricultura y la producción de alimentos ocupan el primer lugar. En base a esto, el Programa de Desarrollo Agropecuario del municipio de Sancti Spíritus (2015-2030) se basa en la planificación y ejecución de 29 subprogramas que tienen como propósito, el cumplimiento de los Lineamientos de la Política Económica y Social del Partido y la Revolución para el período 2016-2021 (Partido Comunista de Cuba, 2017), y la satisfacción de las necesidades territoriales en esta área.

Según el consenso de los directivos y los expertos entrevistados, el modelo de desarrollo agropecuario que impera en el municipio es híbrido. Por una parte, se refieren a este como modelo intensivo, debido a la implementación y el uso de tecnologías como sistemas de riego, tractores, productos químicos, infraestructura de caminos, viales rurales y almacenes. Por otra parte, exponen criterios propios de un modelo agroecológico, cuando reconocen su convivencia con manifestaciones del movimiento "de campesino a campesino», promovido por la Asociación Nacional de Agricultores Pequeños.

Como cabecera provincial, en Sancti Spíritus se localizan las principales entidades empresariales del Ministerio de la Agricultura y las correspondientes a este nivel. En las entidades de alcance municipal se encuentran las dedicadas a la producción, asistencia técnica, servicios y logística (dirección y control). Otras 
entidades, aunque ubicadas espacialmente aquí, responden a intereses provinciales y nacionales, motivo por el cual su accionar no está intencionado directamente desde la gestión municipal, sino también desde otras instancias. La producción de arroz y de tabaco se realiza en las tierras de la base productiva atendidas por las empresas, por lo que las entidades encargadas de coordinar, asistir, beneficiar y comercializar estas producciones no poseen tierras en el territorio, como el caso de la Empresa Agroindustrial de Granos Sur del Jíbaro.

Figura 2. Estructura del sistema del Ministerio de la Agricultura en Sancti Spíritus
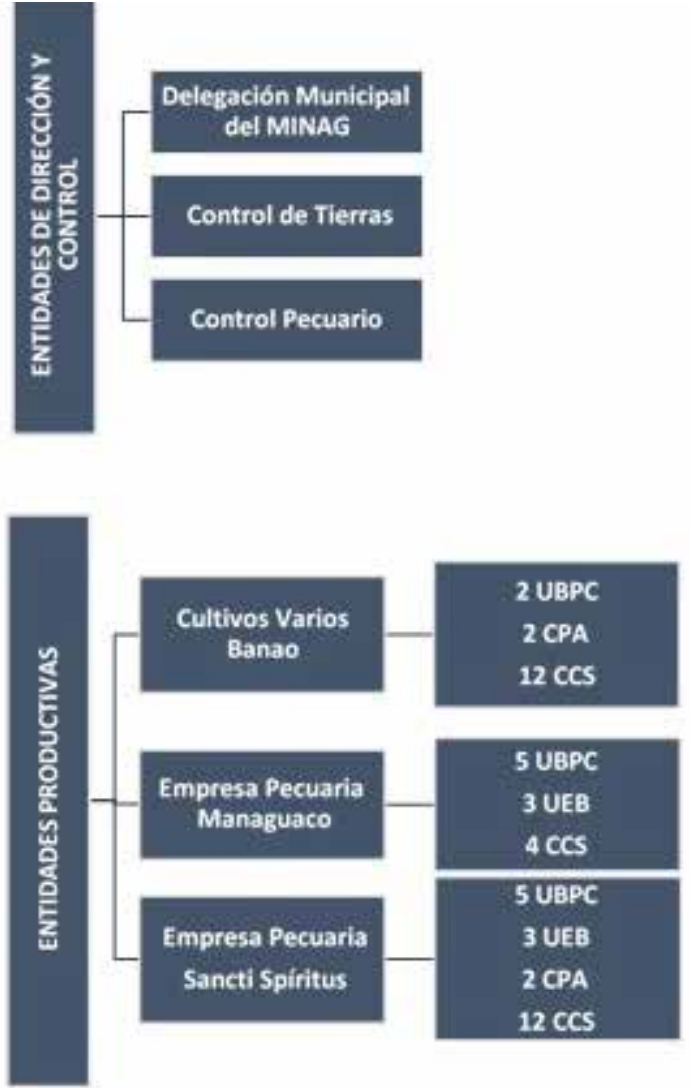

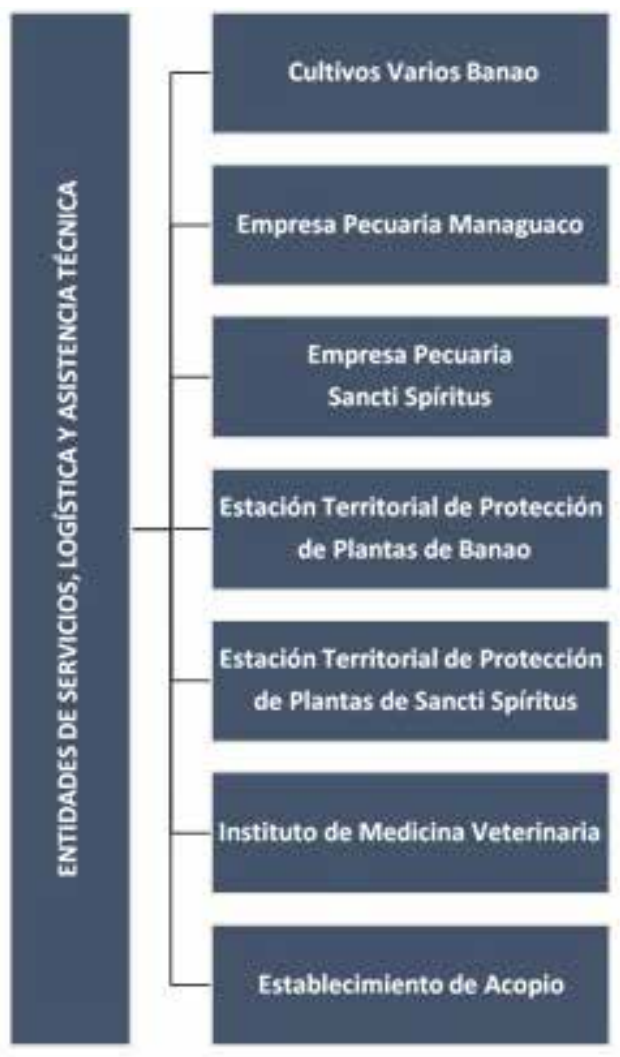

Nota: MINAG: Ministerio de la Agricultura, UBPC: Unidad Básica de Producción Cooperativa, CPA: Cooperativa de Producción Agropecuaria, CCS: Cooperativa de Créditos y Servicios, UEB: Unidad Empresarial de Base.

Fuente: Elaboración propia

Fundamentalmente, la producción agropecuaria espirituana se basa en la actividad agrícola y ganadera, donde los cultivos principales son la caña de azúcar, el tabaco, el café, el arroz, tubérculos y raíces, vegetales y frutales varios. También se explotan los recursos forestales y la ganadería vacuna, porcina y avícola. La producción agropecuaria tiene una alta incidencia en la industria alimentaria a la par que conforma la principal actividad industrial del territorio.

El potencial hidráulico es fundamental para el desarrollo agropecuario a considerar. En el municipio se han construido un total de 21 micropresas con el objetivo de utilizarlas como fuente de abasto para facilitar el riego. Estas obras conforman un volumen de embalse total de $10.58 \mathrm{hm}^{3}$. La provincia 
cuenta con la presa Zaza, cuyo volumen de embalse es 1020 hm $^{3}$; Higuanojo con 24.2 hm $^{3}$; y Banao II con $3.2 \mathrm{hm}^{3}$; lo que suma un total de $1047.6 \mathrm{hm}^{3}$. También existe una reserva de escurrimiento subterráneo que puede ser utilizada para el riego; sin embargo, esta actividad se ve limitada por la carencia de pozos con gasto suficiente. La cuenca de Banao es la única en el territorio que es aprovechada, con un total de 14 pozos. El destino principal de las aguas subterráneas es el abasto a la población y el consumo animal.

A su vez, el municipio tiene una amplia red fluvial, de ahí que también los suelos aluviales sean representativos del territorio. Haciendo una simple correlación entre suelos y núcleos de producción agrícolas, pueden señalarse los Ferralíticos Rojos (caña de azúcar, ganadería y café), los Pardos (área tabacalera y de cultivos varios); los Vertisoles (caña de azúcar, ganadería y cultivos forestales); Pocos evolucionados (forestales y algunas áreas ganaderas), y otros diseminados por todo el territorio con uso variado, donde los Ferralíticos y Aluviales se relacionan con zonas productivas.

El uso sustentable del recurso suelo tiene una importancia fundamental para el mejoramiento de la calidad de vida de los agricultores. Hoy resulta más claro que solo se alcanzará la sostenibilidad agrícola en beneficio de los recursos naturales, la economía y la salud de la población, si se reducen los agroquímicos y se protegen los valores naturales de aquellos. En relación a este tema se lleva adelante el Programa Provincial de Conservación y Mejoramiento de Suelos, que impacta en los diferentes municipios espirituanos, y que tiene en cuenta las características y problemáticas que afectan a este recurso en el desarrollo agrícola territorial.

La Dirección Provincial de Suelos y Fertilizantes (de conjunto con otras entidades del Ministerio de la Agricultura), el Grupo Empresarial Azucarero AZCUBA, el Ministerio de Ciencia, Tecnología y Medio Ambiente, el Instituto Nacional de Recursos Hidráulicos y otros actores con tenencia de tierras; son los responsables de garantizar que las acciones definidas en el programa sean cumplidas, a partir de su condición de propietarios. Con ello se busca asegurar que el per cápita de tierras agrícolas por habitante no continúe decreciendo.

Sin embargo, no todas las entidades productivas realizan un uso adecuado de este recurso. El mal manejo de los suelos y de los sistemas de cultivo han resultado en una acentuada pérdida de su fertilidad, estructura y materia orgánica; así como en su erosión y contaminación. Estos factores socioambientales han provocado una disminución considerable de la productividad agrícola y un incremento significativo de los costos de producción en el municipio cabecera. En este sentido, cabe mencionar algunas de las causas fundamentales que conllevan a tal situación: los escasos recursos económicos para enfrentar la limpieza del marabú (que en la actualidad se ha convertido en fuente para elaborar carbón vegetal y exportarlo); violaciones en relación con el objeto social de la tenencia de tierras; y limitaciones para la construcción de fuentes que permitan el abastecimiento de agua y su electrificación.

En cuanto al manejo de los suelos, se percibe 
una correcta rotación de los cultivos; pese a ello, no están generalizadas las medidas para su conservación y mejoramiento, condiciones que limitan considerablemente sus rendimientos. Por la incidencia del Proyecto de Innovación Agropecuaria Local, en el territorio se han logrado incorporar nuevas prácticas de manejo que contribuyen a mejorar las áreas de producción, promoviéndose su diversificación y la utilización de los abonos orgánicos. Esta propuesta impacta favorablemente en la calidad de los productos (sanos), así como en la economía de los cooperativistas y sus familias. Los agricultores son las personas claves para decidir sobre el uso y manejo de la tierra, por ende, sus conocimientos y demandas deben constituir el insumo idóneo para la creación de un programa de manejo y conservación de suelos y agua.

Teniendo en cuenta la densidad poblacional del municipio de Sancti Spíritus, el sector de la agricultura debe fortalecer sus producciones sin perder de vista que la oferta no satisface la demanda, expresada en la distribución de $5.4 \mathrm{~m}^{3} / \mathrm{hab}$. A pesar de las limitaciones de la oferta en relación al consumo, los decisores del sector agrario no pretenden incrementar los organopónicos ni los sistemas semiprotegidos, sino mantener la base productiva con un alto nivel organizativo y de explotación, mediante la permanente actualización técnica y la capacitación de los productores. Este aspecto constituye una tarea de suma prioridad para alcanzar rendimientos productivos superiores y el incremento de la cultura agraria, alimentaria y ambiental.

Actualmente, las entidades del sector llevan adelante un grupo de proyectos en aras de perfeccionar el sistema agroproductivo, a partir de la creación y el fortalecimiento de capacidades innovadoras en función del desarrollo económico local. Dentro de ellos, se destacan por los objetivos que persiguen y el alcance de sus resultados, la Asociación Nacional de Agricultores Pequeños, la Asociación Cubana de Técnicos Agrícolas Forestales, la Escuela de Capacitación de la Agricultura, la Escuela de Ganadería, el Ministerio de Ciencia, Tecnología y Medio Ambiente, la Universidad de Sancti Spíritus y el Ministerio de la Agricultura.

La Asociación Nacional de Agricultores Pequeños lidera estequehacercon una cartera de proyectos vinculados a la productividad agrícola, y al fomento de la cultura ambiental en las comunidades o escenarios en los que inciden; los que además orientan el empleo de las energías renovables, específicamente los biodigestores de policloruro de vinilo (conocido como PVC), recurso sumamente económico. Los proyectos no solo permiten probar tecnologías e innovar en la gestión del desarrollo local, sino que también promueven la creación de capacidades que garanticen su sostenibilidad.

Este es justamente uno de los desafíos más importantes en cuanto a la inserción de las tecnologías como alternativa para el desarrollo en contextos locales, pues considerar la sostenibilidad desde la perspectiva de su integración en el proceso de desarrollo local no es únicamente atender a la sostenibilidad de la infraestructura (equipos, conectividad, personal), sino del proyecto que va a mejorar la educación, la salud, la gobernabilidad, las posibilidades de ingresos, etc. Al respecto, comentó el delegado del Ministerio de la Agricultura en el municipio: 
Los campesinos hoy están mejor informados, incluso para enfrentar los proyectos internacionales, pero esto aún no se ha generalizado, no existe esta cultura, una comunicación que garantice lo que algunos ya han alcanzado y que el resto debe y pudiera aprovechar. (R. Plasencia, comunicación personal, 23 de diciembre de 2019)

La participación de mujeres y hombres en estas iniciativas significa una oportunidad para reflexionar sobre los factores que inciden en la desigualdad social, la invisibilidad de la mujer en el contexto rural y las barreras de género (Campos-Gómez et al., 2018). En tal sentido, se hace alusión al desarrollo local y al fortalecimiento de esTe capital social como recurso sustancial para la gestión de dicho desarrollo; expectativas que se alcanzarán a largo plazo, pues el tiempo de duración del proyecto solo permite promover la reflexión e incorporar acciones concretas hacia la igualdad de oportunidades, en un marco privilegiado de beneficios sociales, técnicos y financieros.

El nuevo modelo económico cubano impulsa la capacitación para potenciar los resultados productivos, económicos, sociales y ambientales del sector. Al respecto, R. Plasencia opinó que «es necesario fortalecer los espacios de formación y capacitación, ya sea en las asambleas, las fincas de los campesinos o en la empresa; sin dudas, estos espacios permitirán enriquecer los conocimientos de todos en aras de perfeccionar nuestro desempeño» (comunicación personal, 23 de diciembre de 2019).

En tal sentido, el Ministerio de la Agricultura traza anualmente un plan de capacitación desde sus entidades territoriales, a partir de las necesidades y demandas de sus bases productivas. Este se ejecuta tanto en la Escuela de Capacitación de la Agricultura $u$ otras instituciones y organizaciones del municipio, como en la Asociación Nacional de Agricultores Pequeños, la Universidad de Sancti Spíritus o el Proyecto de Innovación Agropecuaria Local. Una de sus líneas fundamentales es el tema de la cultura cooperativa, pues ella no se percibe como hecho de socialización. Los principios del cooperativismo están asociados a valores espirituales, de identidad, que son necesarios fortificar. En la entrevista realizada al coordinador de capacitación del Ministerio de la Agricultura y de la Asociación Cubana de Técnicos Agrícolas Forestales este consideró:

Las cooperativas son autónomas y, sin embargo, muchos cooperativistas no están preparados para asumir todo lo que esto implica, todavía ellos sienten que son hijos de la empresa. Es necesario enseñarlos a trabajar con independencia, porque esa es la esencia del cooperativismo. (E. Rodríguez, comunicación personal, 12 de enero de 2020)

\section{La capacidad social de innovación, un componente medular para la implementación del SIAL}

Concebir e impulsar la innovación en el ámbito local constituye una tarea de primer orden (Núñez et al., 2015); pese a ello, resulta alarmante que la inversión económica en el desarrollo de dicha actividad continúe siendo insuficiente dentro del sector agropecuario espirituano, en contraste con la capacidad renovadora del municipio, que ha logrado aplicar numerosas experiencias. En Sancti Spíritus, tres polos productivos han recibido 
recursos diferenciados en función de ese objetivo: Banao; las seis Cooperativas de Producción Agropecuaria de Cabaiguán y la Empresa Valle del Caonao, específicamente en Batey Colorado.

En este sentido, con dichas prácticas se han logrado avances significativos en el desarrollo de tecnologías adecuadas para mejorar la productividad agropecuaria en sistemas sostenibles. No obstante, solo en algunos casos estas innovaciones logran extenderse y replicarse, debido a la poca integración de los componentes de los sistemas agrícolas; y a las prácticas comunicativas que caracterizan esta relación, donde prima la verticalidad, no así el diálogo de saberes y la retroalimentación de experiencias.

Existen actores potenciales que buscan impulsar el movimiento innovador $y$, desde sus aportes prácticos, convertir los obstáculos en oportunidades. La Asociación Nacional de Innovadores y Racionalizadores, los centros de investigación (Ministerio de Ciencia, Tecnología y Medio Ambiente, Universidad de Sancti Spíritus, Biotecnología, etc.) y el fórum, término que designa la estructura y la fuerza organizativa que atiende la innovación dentro del sistema del Poder Popular Municipal, constituyen los principales ejemplos de ello.

Como movimiento político, el fórum es guiado por el Partido Comunista de Cuba y presidido por el gobierno en el territorio. Integra instituciones, organizaciones, centros y grupos de individuos en función de la ciencia y la técnica; y promueve la búsqueda y la aplicación de soluciones concretas a las necesidades imperantes. Se organiza en grupos de cooperación tecnológica, los que permiten el reconocimiento y alianza de sus miembros en el diseño de alternativas estratégicas, principalmente en los temas: biotecnología e industria farmacéutica; salud, energía y alimentación; tecnología, piezas, equipos y medios para los servicios; la refrigeración en el turismo; fabricación y recuperación de piezas de repuesto; equipos, tecnologías para el transporte, la construcción y la vivienda; sector estudiantil; sector de la defensa; organización, gestión y dirección económicas; entre otros.

Dichos grupos de cooperación constituyen catalizadores para el desarrollo de la ciencia, la tecnología y la innovación, pues contribuyen a potenciar y socializar las prácticas innovadoras en las temáticas priorizadas para el trabajo del fórum, teniendo en cuenta las demandas del territorio. A pesar de la organización de ese movimiento, la definición de las prioridades y la clara pertinencia de sus resultados, aún existen grandes limitaciones para una adecuada gestión del conocimiento en función de las problemáticas; causas asociadas fundamentalmente a la moderada sensibilización y compromiso de los directivos con esta actividad.

En la entrevista realizada al delegado del Ministerio de la Agricultura en el municipio se explicaron las acciones realizadas para potenciar la innovación en el sector, las cuales resultan reiteradas y aisladas; también se reconoció la ausencia de una estrategia municipal que potencie la innovación de forma sistémica:

Hace unos años eso estaba un poco más organizado, y aunque existe el movimiento de la Asociación Nacional 
de Innovadores y Racionalizadores, y el fórum, que impulsa bastante este tema, creo que pudiera hacerse mucho más, sobre todo en cuanto a la articulación de los innovadores. (R. Plasencia, comunicación personal, 23 de diciembre de 2019)

El Proyecto de Innovación Agropecuaria Local ha concebido el SIAL como propuesta para potenciar y articular los esfuerzos en pro de la innovación, pero su institucionalización ha presentado múltiples desafíos. Aunque se percibe un reconocimiento de la pertinencia de la propuesta, desde el gobierno no se ha logrado incidir de forma integrada en el municipio, lo cual ha limitado los espacios de concertación entre los grupos de innovación agropecuaria local ya creados y los actores locales vinculados a la agricultura. Lograr instaurar el SIAL en armonía con el contexto requiere fortalecer, ante todo, los espacios concebidos para su gestión (plataformas multiactorales de gestión, grupos de innovación agropecuaria local); sin embargo, es vital sensibilizar a los actores locales en cuanto a los beneficios y oportunidades que ofrece la propuesta para el desarrollo integral del territorio. En la entrevista a la jefa del Departamento de Ciencia y Técnica del Poder Popular Municipal, se comentaban las razones por las que no se ha logrado impulsar el SIAL armónicamente:

No hemos podido implementar las plataformas multiactorales de gestión por falta de compromiso y sensibilización, no solo con el desarrollo agropecuario, sino también con el desarrollo local. El personal vinculado al desarrollo local es muy inestable y en algunos casos, les falta visión estratégica. Aquí, en el gobierno, no tenemos un grupo de desarrollo local, solo podemos mencionar acciones puntuales en este tema y así es difícil avanzar. (V. Quintero, comunicación personal, 26 de enero de 2020)

Paradójicamente, como resultados de las acciones del Proyecto de Innovación Agropecuaria Local en el territorio se destaca la presencia de 42 fincas diversificadas, las cuales son consideradas muestras de confianza. Las fincas donde funcionan los grupos de innovación agropecuaria local han incrementado el número de especies, variedades, accesiones y clones, así como la producción de cultivos como fríjol, boniato, arroz, yuca y tomate en un $8 \%$.

En la etapa se han consolidado las alianzas con el proyecto Biomas-Cuba, el Programa de Desarrollo Local; la Asociación Cubana de Técnicos Agrícolas Forestales, la Asociación Nacional de Agricultores Pequeños, la Federación de Mujeres Cubanas, el Centro de Reflexión y Diálogo Oscar Arnulfo Romero; todo ello enfocado a los procesos en la base y a la transversalización de género y juventud.

No obstante, la carencia de un sistema integrado de gestión de la innovación, como el que se ilustra en la figura 3, no permite planificar, organizar, controlar, estudiar y potenciar el desarrollo agropecuario en el municipio, orientado a empoderar diferentes grupos sociales mediante la concientización, la sostenibilidad y la diversidad productiva, desde la formación de capacidades innovadoras que vayan de la creatividad hasta la resolución de las dificultades con los recursos y la gestión 
locales. Promover la horizontalidad de los procesos permitirá impulsar las iniciativas y las acciones propuestas hacia nuevos logros, y facilitar, a su vez, la participación proactiva y el compromiso colectivo.

Figura 3. Diagrama del Sistema de Innovación Agropecuaria Local

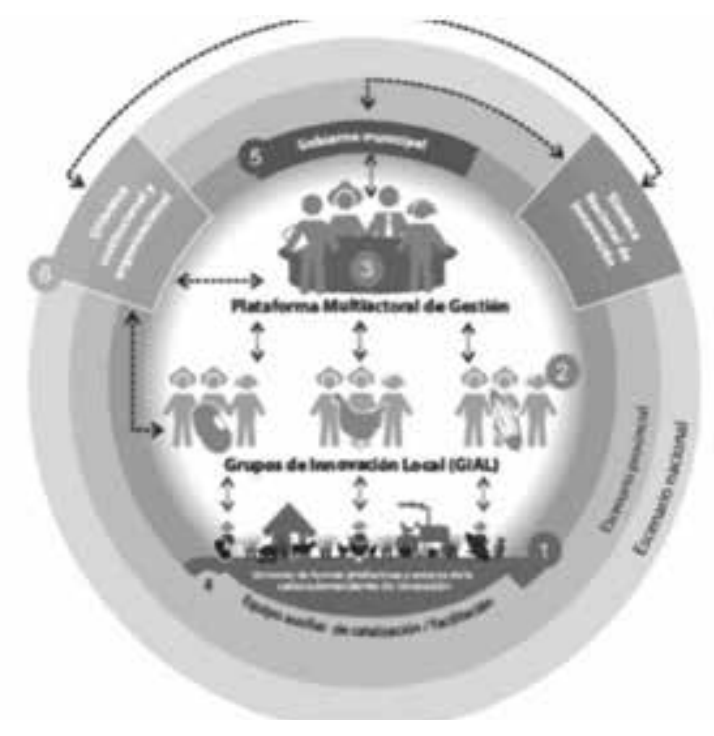

Fuente: Ortiz et al. (2016).

\section{Propuesta de acciones para la implementación del SIAL en Sancti Spíritus}

Teniendo en cuenta que el municipio de Sancti Spíritus muestra aciertos y limitaciones en la implementación del SIAL, se hace pertinente el diseño de un sistema de acciones que promuevan su institucionalidad y reconocimiento como espacio ideal de concertación entre los actores locales. Las acciones deberán ser desarrolladas por un grupo de facilitación, de conjunto con otros actores que integran las cadenas agroproductivas, mediante una relación sustentada en el diálogo, la participación y la retroalimentación de saberes teóricos y prácticos. De ello se deriva la siguiente propuesta de acciones:

Tabla 1. Plan de acción para la institucionalización del SIAL en el municipio Sancti Spíritus

\begin{tabular}{|c|c|c|c|}
\hline \multicolumn{4}{|c|}{ I. Formar el grupo auxiliar de facilitación/catalización } \\
\hline Acciones & Objetivo & Participantes & Responsables \\
\hline $\begin{array}{l}\text { Concertar despachos con } \\
\text { líderes políticos y de gobierno. }\end{array}$ & $\begin{array}{l}\text { Sensibilizar a los directivos y } \\
\text { líderes políticos para } \\
\text { desarrollar integralmente el } \\
\text { SIAL. }\end{array}$ & $\begin{array}{l}\text { Líderes políticos y de } \\
\text { gobierno, } \\
\text { integrantes de PIAL. }\end{array}$ & $\begin{array}{l}\text { Equipo } \\
\text { coordinador del } \\
\text { PIAL. }\end{array}$ \\
\hline $\begin{array}{l}\text { Reunión de intercambio con } \\
\text { representantes del grupo de } \\
\text { desarrollo local, el Consejo de } \\
\text { Administración Municipal y la } \\
\text { PMG. }\end{array}$ & $\begin{array}{l}\text { Facilitar el debate y la } \\
\text { contextualización de un } \\
\text { diplomado sobre las } \\
\text { particularidades del SIAL y } \\
\text { las demandas del territorio. }\end{array}$ & $\begin{array}{l}\text { Representantes del } \\
\text { grupo de desarrollo } \\
\text { local, el Consejo de } \\
\text { Administración } \\
\text { Municipal y la PMG. }\end{array}$ & $\begin{array}{l}\text { Equipo } \\
\text { coordinador del } \\
\text { PIAL. }\end{array}$ \\
\hline
\end{tabular}




\begin{tabular}{|c|c|c|c|}
\hline $\begin{array}{l}\text { Socialización de la propuesta } \\
\text { final del diplomado y el } \\
\text { cronograma de ejecución con } \\
\text { los organismos implicados. }\end{array}$ & $\begin{array}{l}\text { Consolidar el compromiso } \\
\text { con la propuesta del } \\
\text { diplomado y garantizar la } \\
\text { participación de los actores } \\
\text { convocados en él. }\end{array}$ & $\begin{array}{l}\text { Representantes del } \\
\text { grupo de desarrollo } \\
\text { local, el Consejo de } \\
\text { Administración } \\
\text { Municipal, la PMG, } \\
\text { el MINAG, el CITMA, } \\
\text { la ANAP y la UNISS. }\end{array}$ & $\begin{array}{l}\text { Equipo } \\
\text { coordinador del } \\
\text { PIAL. }\end{array}$ \\
\hline $\begin{array}{l}\text { Conformar el equipo de } \\
\text { coordinación del diplomado. }\end{array}$ & $\begin{array}{l}\text { Promover la interconexión } \\
\text { entre los organismos } \\
\text { implicados. }\end{array}$ & $\begin{array}{l}\text { Representantes del } \\
\text { Grupo de Desarrollo } \\
\text { Local, el Consejo de } \\
\text { Administración } \\
\text { Municipal, la PMG, } \\
\text { el MINAG, el CITMA, } \\
\text { la ANAP y la UNISS. }\end{array}$ & $\begin{array}{l}\text { Equipo } \\
\text { coordinador } \\
\text { PIAL. }\end{array}$ \\
\hline $\begin{array}{l}\text { Seguimiento y evaluación por } \\
\text { parte del equipo de } \\
\text { coordinación del diplomado. }\end{array}$ & $\begin{array}{l}\text { Lograr un acompañamiento } \\
\text { sistemático en las acciones. }\end{array}$ & $\begin{array}{l}\text { Diplomantes } \\
\text { equipo } \\
\text { coordinación. }\end{array}$ & $\begin{array}{l}\text { Equipo de } \\
\text { coordinación del } \\
\text { diplomado. }\end{array}$ \\
\hline
\end{tabular}

\begin{tabular}{|c|c|c|c|}
\hline \multicolumn{4}{|c|}{ II. Realizar actividades generadoras de zonas de aprendizajes } \\
\hline Acciones & Objetivo & Participantes & Responsables \\
\hline $\begin{array}{l}\text { Creación de círculos de interés } \\
\text { para niños y jóvenes de las } \\
\text { comunidades. }\end{array}$ & $\begin{array}{l}\text { Promover en los niños y } \\
\text { jóvenes el conocimiento } \\
\text { sobre los procesos de } \\
\text { innovación y su importancia } \\
\text { para el desarrollo } \\
\text { comunitario. }\end{array}$ & $\begin{array}{l}\text { Niños y jóvenes de } \\
\text { las comunidades } \\
\text { rurales. }\end{array}$ & $\begin{array}{lr}\text { Líderes de la } \\
\text { comunidad } & \text { e } \\
\text { integrantes del } \\
\text { PIAL. }\end{array}$ \\
\hline $\begin{array}{l}\text { Talleres de formación } \\
\text { vocacional para niños y } \\
\text { jóvenes de las comunidades. }\end{array}$ & $\begin{array}{l}\text { Cultivar el interés y la } \\
\text { vocación hacia los procesos } \\
\text { productivos locales. }\end{array}$ & $\begin{array}{l}\text { Niños y jóvenes de } \\
\text { las comunidades } \\
\text { rurales. }\end{array}$ & $\begin{array}{l}\text { Líderes de la } \\
\text { comunidad } \mathrm{e} \\
\text { integrantes del } \\
\text { PIAL. }\end{array}$ \\
\hline $\begin{array}{l}\text { Eventos de ciencia y técnica } \\
\text { en las comunidades y } \\
\text { entidades productivas. }\end{array}$ & $\begin{array}{l}\text { Reconocer y promover el } \\
\text { desarrollo científico de los } \\
\text { actores a nivel local. }\end{array}$ & $\begin{array}{l}\text { Productores líderes } \\
\text { y miembros de la } \\
\text { comunidad. }\end{array}$ & $\begin{array}{l}\text { Equipo } \\
\text { coordinador del } \\
\text { PIAL y la UNISS. }\end{array}$ \\
\hline $\begin{array}{l}\text { Convivencias de estudiantes } \\
\text { universitarios en comunidades } \\
\text { rurales. }\end{array}$ & $\begin{array}{l}\text { Promover el intercambio de } \\
\text { conocimientos entre } \\
\text { campesinos, estudiantes y }\end{array}$ & $\begin{array}{l}\text { Estudiantes y } \\
\text { profesores de las } \\
\text { carreras vinculadas }\end{array}$ & $\begin{array}{l}\text { Equipo } \\
\text { coordinador del } \\
\text { PIAL y la UNISS. }\end{array}$ \\
\hline $\begin{array}{l}\text { Festivales de innovación como } \\
\text { herramienta para diseminar } \\
\text { productos de la innovación a } \\
\text { escala local. }\end{array}$ & $\begin{array}{lcc}\text { Desarrollar } & \text { capacidades } & \text { y } \\
\text { habilidades } & \text { para la } \\
\text { innovación } & \text { en los } \\
\text { productores y otros actores. }\end{array}$ & $\begin{array}{l}\text { Productores líderes } \\
\text { y miembros de la } \\
\text { comunidad. }\end{array}$ & $\begin{array}{lr}\text { Líderes de la } \\
\text { comunidad } & \text { e } \\
\text { integrantes del } \\
\text { PIAL. }\end{array}$ \\
\hline $\begin{array}{l}\text { Creación de grupos de } \\
\text { facilitación para el desarrollo } \\
\text { agropecuario local. }\end{array}$ & $\begin{array}{l}\text { Garantizar la sostenibilidad y } \\
\text { el desarrollo del SIAL. }\end{array}$ & $\begin{array}{l}\text { Productores líderes, } \\
\text { miembros de la } \\
\text { comunidad e } \\
\text { integrantes del PIAL. }\end{array}$ & $\begin{array}{l}\text { La comunidad e } \\
\text { integrantes del } \\
\text { PIAL. }\end{array}$ \\
\hline $\begin{array}{l}\text { Creación de espacios } \\
\text { frecuentes para la } \\
\text { socialización y el intercambio } \\
\text { de experiencias y } \\
\text { conocimientos sobre prácticas } \\
\text { agrícolas. }\end{array}$ & $\begin{array}{l}\text { Facilitar el intercambio de } \\
\text { saberes y experiencias entre } \\
\text { productores y comunidad. }\end{array}$ & $\begin{array}{l}\text { Productores líderes, } \\
\text { miembros de la } \\
\text { comunidad e } \\
\text { integrantes del PIAL. }\end{array}$ & $\begin{array}{l}\text { Productores } \\
\text { líderes y equipo } \\
\text { coordinador del } \\
\text { PIAL. }\end{array}$ \\
\hline
\end{tabular}


III. Definir el grupo de innovación agropecuaria local, los consejos populares participantes y la plataforma multiactoral de gestión. Facilitar su funcionamiento

\begin{tabular}{|c|c|c|c|}
\hline Acciones & Objetivo & Participantes & Responsables \\
\hline $\begin{array}{l}\text { Talleres para la sensibilización } \\
\text { de los actores locales. }\end{array}$ & $\begin{array}{l}\text { Lograr un mayor compromiso } \\
\text { y participación de los actores. }\end{array}$ & $\begin{array}{l}\text { Actores locales e } \\
\text { integrantes del PIAL. }\end{array}$ & $\begin{array}{l}\text { Equipo } \\
\text { coordinador } \\
\text { del PIAL. }\end{array}$ \\
\hline $\begin{array}{l}\text { Diagnóstico participativo de la } \\
\text { comunidad. }\end{array}$ & $\begin{array}{l}\text { Identificar las fortalezas y las } \\
\text { debilidades de la comunidad } \\
\text { para el desarrollo del SIAL. }\end{array}$ & $\begin{array}{l}\text { Miembros de la } \\
\text { comunidad } \\
\text { integrantes del PIAL. }\end{array}$ & $\begin{array}{l}\text { Equipo } \\
\text { coordinador } \\
\text { del PIAL. }\end{array}$ \\
\hline Creación de un grupo gestor. & $\begin{array}{l}\text { Conformar la estructura } \\
\text { responsable de acompañar } \\
\text { sistemáticamente } \\
\text { desarrollo del SIAL. }\end{array}$ & $\begin{array}{l}\text { Productores líderes, } \\
\text { miembros de la } \\
\text { comunidad e } \\
\text { integrantes del PIAL. } \\
\end{array}$ & $\begin{array}{l}\text { Equipo } \\
\text { coordinador } \\
\text { del PIAL. }\end{array}$ \\
\hline \multicolumn{4}{|c|}{ IV. Conciliar las demandas locales con las políticas y estrategias municipales de desarrollo agropecuario } \\
\hline Acciones & Objetivo & Participantes & Responsables \\
\hline $\begin{array}{l}\text { Identificación de espacios } \\
\text { colaboración dentro del sistema } \\
\text { de trabajo del Consejo de } \\
\text { Administración Municipal, el } \\
\text { MINAG y otras entidades } \\
\text { implicadas. }\end{array}$ & $\begin{array}{l}\text { Lograr la inclusión del SIAL } \\
\text { en los sistemas de trabajo de } \\
\text { las entidades implicadas. }\end{array}$ & $\begin{array}{l}\text { Representantes de } \\
\text { los gobiernos } \\
\text { municipales, equipo } \\
\text { coordinador del PIAL. }\end{array}$ & $\begin{array}{l}\text { Equipo } \\
\text { coordinador } \\
\text { del PIAL. }\end{array}$ \\
\hline $\begin{array}{l}\text { Identificación de las demandas } \\
\text { en relación con las estrategias } \\
\text { de desarrollo municipales. }\end{array}$ & $\begin{array}{l}\text { Promover el intercambio y el } \\
\text { análisis en torno a la } \\
\text { correspondencia entre las } \\
\text { estrategias de desarrollo } \\
\text { municipales y las demandas } \\
\text { de la agricultura. }\end{array}$ & $\begin{array}{l}\text { Representantes de } \\
\text { los gobiernos } \\
\text { municipales y } \\
\text { miembros de la } \\
\text { comunidad. }\end{array}$ & $\begin{array}{l}\text { Equipo } \\
\text { coordinador } \\
\text { del PIAL. }\end{array}$ \\
\hline $\begin{array}{l}\text { Socialización de buenas } \\
\text { prácticas vinculadas a los } \\
\text { procesos innovativos locales. }\end{array}$ & $\begin{array}{l}\text { Promover la participación } \\
\text { activa y comprometida de } \\
\text { líderes políticos con el SIAL }\end{array}$ & $\begin{array}{l}\text { Productores líderes, } \\
\text { miembros de la } \\
\text { comunidad } \\
\text { integrantes del PIAL. }\end{array}$ & $\begin{array}{l}\text { Equipo } \\
\text { coordinador } \\
\text { del PIAL. }\end{array}$ \\
\hline $\begin{array}{l}\text { Concertación de espacios en los } \\
\text { medios de comunicación } \\
\text { masiva para la socialización de } \\
\text { los resultados de la } \\
\text { sistematización de experiencias } \\
\text { sobre los procesos innovativos } \\
\text { locales y el SIAL. }\end{array}$ & $\begin{array}{l}\text { Difundir } r \text { conocimientos } \\
\text { sobre los procesos } \\
\text { innovativos locales y el SIAL. }\end{array}$ & $\begin{array}{lr}\begin{array}{l}\text { Miembros de la } \\
\text { comunidad, }\end{array} \\
\text { productores, } \\
\text { medios } \\
\begin{array}{l}\text { comunicación. } \\
\text { de }\end{array}\end{array}$ & $\begin{array}{l}\text { Equipo } \\
\text { coordinador } \\
\text { del PIAL }\end{array}$ \\
\hline
\end{tabular}

Nota: PIAL: Proyecto de Innovación Agropecuaria Local, PMG: Plataformas Multiactorales de Gestión, ANAP: Asociación Nacional de Agricultores Pequeños, MINAG: Ministerio de la Agricultura, CITMA: Ministerio de Ciencia, Tecnología y Medio Ambiente y UNISS: Universidad de Sancti Spíritus.

\section{Fuente: Elaboración propia}

Para la implementación del sistema de acciones se sugiere emplear la metodología de la educación popular, vista desde su «apuesta pedagógica en la orientación de procesos de construcción del conocimiento y de socialización que incluye prácticas, saberes, dinámicas socioculturales e interacciones para transformar la sociedad desde ciudadanías incluyentes y resistencias culturales» (Guelman et al., 2018, p. 10); 
pues esta fomenta una ética liberadora que valoriza al ser humano como sujeto de conocimiento y transformación, desde una relación horizontal con el equipo de facilitación.

Como reconocen Pérez et al. (2019), «esta forma de participación y construcción colectiva pretende promover el empoderamiento de la gente, al ser parte de la solución del problema» (p. 18); lo cual representa una palanca de cambio para el desarrollo exitoso del SIAL. De manera específica, se promueve un nuevo sistema de relaciones con sentido participativo, dialógico y horizontal, desde los actores implicados, con ellos y para ellos. Al mismo tiempo, genera aprendizajes y alianzas estratégicas para el empoderamiento de los sujetos locales y comunitarios.

\section{CONCLUSIONES}

En Sancti Spíritus, la aplicación de numerosas experiencias innovadoras en el sector agropecuario evidencia una capacidad renovadora y un panorama esperanzador para el desarrollo de este. Sin embargo, aún existen grandes limitaciones para el desarrollo de un movimiento innovador articulado y eficiente.
Aun cuando el Proyecto de Innovación Agropecuaria Local constituye una plaza fuerte para el estímulo a la innovación agropecuaria, que ha concebido el SIAL como propuesta para potenciar y articular los esfuerzos en pro de la innovación, no se ha logrado impulsar armónicamente la iniciativa, debido al escaso compromiso y sensibilización de algunos actores locales y la falta de articulación entre estos, lo cual impide fortalecer los espacios diseñados para su gestión (plataformas multiactorales de gestión, grupos de innovación agropecuaria local).

El sistema de acciones propuesto en la presente investigación permite promover y dinamizar la institucionalización del SIAL y su reconocimiento como espacio ideal de concertación entre los actores locales, al concebirse bajo la convicción de que los productores son los verdaderos protagonistas del desarrollo en el sector. Como su enfoque sistémico se basa en el fortalecimiento del diálogo, la participación y la retroalimentación de saberes teóricos y prácticos, su implementación puede constituir un referente para la integración de los actores del sector agropecuario en el municipio.

\section{REFERENCIAS BIBLIOGRÁFICAS}

Arzola, L., Fis, Y. y González, K. (2017). Estudio social del Sistema de Innovación Agropecuario Local en la provincia de Ciego de Ávila. Universidad \& Ciencia, 6(Especial), 229-243. https://bit.ly/3q1HI79

Campos-Gómez, M., Miranda-Tortoló, T., Oropeza-Casanova, T., Plana-Ramos, D., SánchezCárdenas, S. y Bover-Felices, K. (2018). Experiencia de género en el Programa de Innovación Agropecuaria Local en la provincia de Matanzas, Cuba. Pastos y Forrajes, 41(2), 151-156. https://bit.ly/2ZYk3o4 
Fajardo, L., Figueras, D., Santos, I., Roque, Y., Pérez, A., Toledo, G., Peña, R. y Díaz, O. (2013). Innovación social y desarrollo local: Documentación y sistematización de experiencias. Ediciones Luminaria.

Garcés, G., Castañeda, P. y Estrada, A. (2020). Arreglo para la producción artesanal de ladrillo en Bayamo. En A. T. Alcázar (Coord.), Arreglos productivos locales en Cuba: Experiencias desde GUCID y PIAL. Editorial UH.

Guelman, A., Cabaluz, F. y Salazar, M. (Coord.). (2018). Educación popular y metodologías críticas en América Latina y el Caribe: corrientes emancipatorias para la educación pública del siglo XXI. (1ra ed.). CLACSO. https://bit.ly/3uD6dWe

Hernández, R., Fernández, C. y Baptista, M. P. (2014). Metodología de la investigación (6ª ed.). Mac Graw Hill. https://bit.ly/2Kl8XFb

Núñez, J., Armas, I., Alcázar, A. y Figueroa, G. (2015). Educación superior, innovación y desarrollo local: experiencias en Cuba. Congreso Universidad, 4(3), 228-248. https://bit.ly/3bOVq2s

Núñez, J. y Alcázar, A. (Coord.). (2016). Universidad y desarrollo local: contribuciones latinoamericanas. Unión de Universidades de América Latina y el Caribe- Editorial Félix varela. https://bit.ly/3r0a10Y

Oficina Nacional de Estadísticas e Información. (2019). Anuario estadístico de Cuba 2018. http:// bit.ly/3vdGd46

Ortiz, R., Angarica, L., Acosta, R. y Guevara, F. (2016). El contexto y su efecto en las salidas de un proyecto de innovación agropecuaria. Cultivos tropicales, 37(2), 141-148. https://bit. Iy/3loWv5E

Partido Comunista de Cuba. (2017). Lineamientos de la política económica y social del partido y la revolución para el período 2016-2021 https://cutt.ly/zyKnCAB

Pérez, C., Paredes, R. y Pérez, J. J. (2019). Organización y participación popular en Cuba: aportes desde el grupo comunitario de educación ambiental. Trabalho necessário, 17(34), 16-41. https://bit.ly/2ZWrLin

Romero, M. I., Ortiz, R. y La O, M. (2018). La gestión del conocimiento en el Sistema de Innovación Agropecuaria Local. Revista Estudios del Desarrollo Social: Cuba y América Latina, 6(3), a14. https://bit.ly/3kxUvHU

Stewart-Santos, E. M., González-Ortiz, M., Soulary-Carracedo, V. S. y Morales-Pérez, M. (2020). Medición del nivel de Desarrollo Local Sostenible en la provincia de Santiago de Cuba. Anuario Facultad de Ciencias Económicas y Empresariales, (Especial 1), 79-90. https://bit. Iy/3cBDWHm 